Article

\title{
Urinary Concentrations of Diisoheptyl Phthalate Biomarkers in Convenience Samples of U.S. Adults in 2000 and 2018-2019
}

\author{
Manori J. Silva *, Lee-Yang Wong, James L. Preau, Ella Samandar, Emmanuela Obi, \\ Antonia M. Calafat and Julianne C. Botelho \\ Division of Laboratory Sciences, National Center for Environmental Health, Centers for Disease Control and \\ Prevention, Atlanta, GA 30341, USA; lyw8@cdc.gov (L.-Y.W.); nzp4@cdc.gov (J.L.P.); evs9@cdc.gov (E.S.); \\ osn8@cdc.gov (E.O.); aic7@cdc.gov (A.M.C.); gur5@cdc.gov (J.C.B.) \\ * Correspondence: zca2@cdc.gov
}

Received: 11 September 2019; Accepted: 4 October 2019; Published: 11 October 2019

check for updates

\begin{abstract}
We know little about the potential health risks from exposure to diisoheptyl phthalate (DiHpP), a plasticizer used in commercial applications. The production of DiHpP ended in the United States in 2010, but DiHpP may still be present in phthalate diester mixtures. To investigate human exposure to $\mathrm{DiHpP}$, we used three oxidative metabolites of DiHpP: Monohydroxyheptyl phthalate (MHHpP), mono-oxoheptylphthalate (MOHpP), and monocarboxyhexyl phthalate (MCHxP) as exposure biomarkers. We analyzed urine collected anonymously in $2000(N=144)$ and 2018-2019 $(N=205)$ from convenience groups of U.S. adults using high-performance liquid chromatography coupled with isotope-dilution high-resolution mass spectrometry. We detected MCHxP in all the samples tested in $2000(\mathrm{GM}=2.01 \mathrm{ng} / \mathrm{mL})$ and 2018-2019 $(\mathrm{GM}=1.31 \mathrm{ng} / \mathrm{mL})$. MHHpP was also detected in $100 \%$ of the $2018-2019$ samples $(\mathrm{GM}=0.59 \mathrm{ng} / \mathrm{mL})$ and $96 \%$ of the 2000 urine samples analyzed $(\mathrm{GM}=0.38 \mathrm{ng} / \mathrm{mL})$. $\mathrm{MOHpP}$ was detected in 57\% $(2018-2019, \mathrm{GM}=0.03 \mathrm{ng} / \mathrm{mL})$ and $92 \%$ (2000, GM $=0.19 \mathrm{ng} / \mathrm{mL}$ ) of samples. The presence of MHHpP, MOHpP, and MCHxP in the 2018-2019 samples suggests recent exposure to DiHpP. Intercorrelations between metabolite concentrations were more significant in samples collected in 2000 than in samples collected in 2018-2019. The differences in urinary metabolite profiles and intercorrelations from samples collected during 2000 and 2018-2019 likely reflects changes in the composition of commercial DiHpP formulations before and after 2010.
\end{abstract}

Keywords: diisoheptyl phthalate; DiHpP plasticizers; exposure; oxidative metabolites

\section{Introduction}

Diisoheptyl phthalate (DiHpP), an isomeric mixture of phthalates with branched and linear seven carbon backbones, is used commercially as a plasticizer in vinyl resins. $\mathrm{DiHpP}$ can be found in automotive, wire, cable, imitation leather, and flooring products [1]. DiHpP production ended in the European Union and United States in 2010 [2]. However, DiHpP may still be present in phthalate diester mixtures [1] that can be used in consumer products in the United States and European Union. As a result, human exposure to DiHpP can occur.

Animal studies suggest potential adverse health effects from exposure to $\mathrm{DiHpP}$ [2-7]. In a 28-day repeated oral DiHpP dose toxicity test in male and female F344 rats, body weight gain was inhibited, but liver and kidney weights increased [8]. In a developmental toxicity study, female rats given $\mathrm{DiHpP}$ by oral gavage on gestational days 6-20 had increased resorptions and reduced fetal weight [7]. Metabolites of phthalates are often used as biomarkers of exposure [9]. To date, no human data on exposure to $\mathrm{DiHpP}$ have been reported. Hence, DiHpP biomonitoring to assess exposure in humans might prove to be valuable. 
Oxidative metabolites form during metabolism of phthalates and are excreted in urine $[10,11]$. Population-based biomonitoring studies use oxidative metabolites of phthalates as biomarkers of exposure [9,12-15]. Monohydroxyheptyl phthalate (MHHpP) and monocarboxyhexyl phthalate (MCHxP), which formed as DiHpP metabolites, were identified in urine of rats dosed with $\mathrm{DiHpP}$ [16]. In our study, we quantified three oxidative metabolites of DiHpP to better understand human exposure to DiHpP.

\section{Materials and Methods}

\subsection{Chemicals and Equipment}

We bought MHHpP, mono-oxoheptyl phthalate (MOHpP), MCHxP (Figure 1), $\mathrm{d}_{4}-\mathrm{MHHpP}$, $\mathrm{d}_{4}-\mathrm{MOHpP}$, and $\mathrm{d}_{4}-\mathrm{MCHxP}$ from ADM Germany $(>95 \%)$. We bought high-performance liquid chromatography (HPLC) grade acetonitrile, water, and methanol (99.8\%) from Honeywell Burdick \& Jackson (Muskegon, MI, USA). $\beta$-glucuronidase (Escherichia coli-K12) was purchased from Roche Biomedical (Mannheim, Germany). All chemicals and reagents were used without further purification.
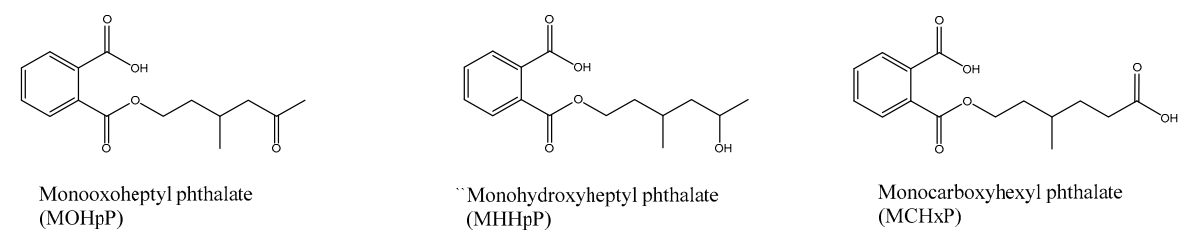

Figure 1. Metabolites used to assess exposure to diisoheptyl phthalate. Only one isomer for each metabolite is shown.

We used a QExactive plus Hybrid Quadrupole-Orbitrap mass spectrometer and Ultimate 3000 high-performance chromatography system (Thermo Fisher Scientific, Waltham, MA, USA) for sample analysis and Xcalibur 2.2 (Thermo Fisher Scientific) for data processing.

\subsection{Subjects}

We collected urine anonymously from demographically diverse groups of U.S. male and female adults from Atlanta, GA, during 2000 and 2018-2019 to study exposure biomarkers to environmental chemicals. No personal information from the subjects was available. Samples were collected between 8:00 a.m. and 5:00 p.m. and were not necessarily first-morning voids. Same donors may have contributed urine in different collection years, during different days, or at different times of day. After collection, samples were stored at $-70{ }^{\circ} \mathrm{C}$ until analysis. The Centers for Disease Control and Prevention (CDC) Institutional Review Board approved the urine collection and analysis. A waiver of informed consent was requested under 45 CFR 46.116(d) general requirements for informed consent.

\subsection{Analytical Method}

We obtained the mass spectra for all three metabolites using analytical standards prepared in acetonitrile and optimized the fragmentation of the precursor ion for each metabolite (Table 1). We adapted published analytical methods for measuring phthalate oxidative metabolites in urine $[17,18]$. Briefly, urine $(0.1 \mathrm{~mL})$ and calibration standards $(0.013-130 \mathrm{ng} / \mathrm{mL})$ were spiked with an internal standard solution (5-10 ng/mL) containing $\mathrm{d}_{4}-\mathrm{MHHpP}, \mathrm{d}_{4}-\mathrm{MOHpP}, \mathrm{d}_{4}-\mathrm{MCHxP}$, and a buffered solution of $\beta$-glucuronidase (Escherichia coli-K12; $25 \mu \mathrm{L}, \mathrm{pH}$ 6.5, $1 \mathrm{M}$ ammonium acetate). The mixture was incubated at $37^{\circ} \mathrm{C}$ for a minimum of $120 \mathrm{~min}[17,18]$. The target analytes in the spiked urine were extracted using on-line solid-phase extraction (Chromolith HighResolution RP-18, $4.6 \mathrm{~mm}$ Guard Cartridge; Merck KGaA, Darmstadt, Germany) and chromatographically resolved using high-performance liquid chromatography (Thermo Scientific Betasil phenyl, $3 \mu \mathrm{m}, 150 \mathrm{~mm} \times 2.1 \mathrm{~mm}$ column) (Figure 2). We then used high-resolution mass spectrometry in negative ion parallel reaction 
mode on a QExactive high-resolution mass spectrometer for quantification of the analytes (Table 1). We used the lowest calibration level $(0.013 \mathrm{ng} / \mathrm{mL})$ as the limit of detection (LOD).

Table 1. Analytical parameters for the quantification of diisoheptyl phthalate metabolites.

\begin{tabular}{ccccc}
\hline Parent Chemical & $\begin{array}{c}\text { Urinary } \\
\text { Metabolite }\end{array}$ & Internal Standard & $\begin{array}{c}\text { MS/MS Scan } \\
\text { (Native) }\end{array}$ & $\begin{array}{c}\text { Collision Energy } \\
\left(\mathbf{e V}^{\text {a }}\right)\end{array}$ \\
\hline Diisoheptyl & MHHpP & $\mathrm{d}_{4}-\mathrm{MHHpP}$ & $279.1 / 121.03$ & 15 \\
phthalate & MOHpP & $\mathrm{d}_{4}-\mathrm{MOHpP}$ & $277.1 / 121.03$ & 16 \\
(DHpP) & MCHxP & $\mathrm{d}_{4}-\mathrm{MCHxP}$ & $293.1 / 145.09$ & 16 \\
\hline
\end{tabular}

${ }^{\text {a }}$ Collision energy applied in QExactive high-resolution mass spectrometer in parallel reaction mode.
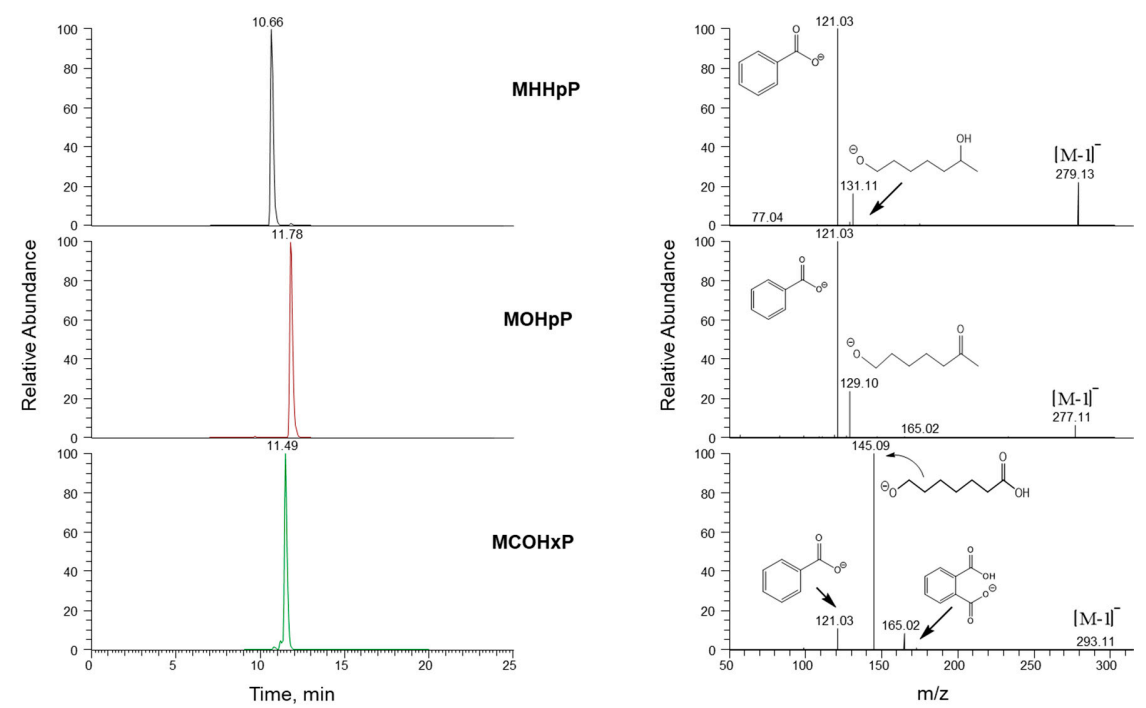

Figure 2. Chromatographic separation and mass spectra of diisoheptyl phthalate metabolites.

We also compared the urinary MHHpP concentrations to the hydroxylated metabolites of dibutyl phthalate, diisobutyl phthalate, and di-2-ethylhexyl phthalate concentrations in human urine reported in the 2015-2016 National Health and Nutrition Examination Survey [19].

We used SAS (version 9.4; SAS Institute Inc., Cary, NC, USA) to perform statistical analyses. For metabolite concentrations below the LOD, we imputed a value equal to the LOD divided by the square root of 2 [20]. Statistical significance was set at Pearson correlation coefficient $(p)<0.05$.

\section{Results and Discussion}

DiHpP has been used in vinyl plastics, including flooring, but no data are available on human exposure. In this proof-of-concept study, we report the urinary concentrations of three oxidative metabolites of DiHpP (MHHpP, MOHpP, and MCHxP). The urine was collected during 2000 and 2018-2019 from convenience samples of U.S. adults not known to be occupationally exposed. We used high-resolution mass spectrometry to resolve analytes from isobaric interferences, which allowed us to quantify the three metabolites at sub-parts per billion concentrations. Because DiHpP consists of multiple isomers with similar physical and chemical properties, metabolites were eluted as broad HPLC peaks with similar mass spectrometric transitions, as reported previously for other isomeric phthalate mixtures [21,22].

Table 2 lists geometric means (GM), select percentile concentrations, and detection frequencies of $\mathrm{DiHpP}$ metabolites in urine. We detected MCHxP in all the urine samples tested. $\mathrm{MHHpP}$ was also detected in the 2018-2019 (100\%) and 2000 (96\%) samples. MOHpP was detected less frequently, at $57 \%$ in the 2018-2019 samples and $92 \%$ in the 2000 samples. The frequent detection of these metabolites in the 2018-2019 samples suggests recent exposure to DiHpP. 
Table 2. Selected percentiles of urinary concentrations ( $95 \% \mathrm{CI})$ of three oxidative metabolites (ng/mL) of diisoheptyl phthalate in a convenience sample of U.S. adults.

\begin{tabular}{|c|c|c|c|c|c|c|c|c|}
\hline \multirow{2}{*}{$\begin{array}{c}\text { Urinary } \\
\text { Metabolite }\end{array}$} & \multirow{2}{*}{ Collection Year } & \multirow{2}{*}{$\mathbf{N}$} & \multicolumn{4}{|c|}{ Percentile } & \multirow{2}{*}{$\begin{array}{c}\text { Geometric } \\
\text { Mean, ng/mL }\end{array}$} & \multirow{2}{*}{$\begin{array}{l}\text { Frequency of } \\
\text { Detection }(\%\end{array}$} \\
\hline & & & 25th & 50 th & 75th & 90th & & \\
\hline \multirow{2}{*}{ МHНpP } & 2018-2019 & 205 & $0.25(0.19,0.29)$ & $0.55(0.4,0.72)$ & $1.71(1.31,2.2)$ & $4.88(3.39,7.07)$ & $0.59(0.5,0.7)$ & 100 \\
\hline & 2000 & 144 & $0.16(0.13,0.21)$ & $0.44(0.34,0.57)$ & $1.04(0.87,1.53)$ & $1.99(1.7,2.4)$ & $0.38(0.31,0.46)$ & 96 \\
\hline \multirow[t]{2}{*}{$\mathrm{MOHpP}$} & 2018-2019 & 205 & $<\mathrm{LOD}^{\mathrm{a}}$ & $\begin{array}{c}0.02(<\mathrm{LOD} \\
0.04)\end{array}$ & $0.1(0.07,0.13)$ & $0.23(0.19,0.3)$ & $\mathrm{NA}^{\mathrm{b}}$ & 57 \\
\hline & 2000 & 144 & $0.07(0.04,0.09)$ & $0.24(0.17,0.32)$ & $0.64(0.51,0.99)$ & $1.29(1.04,1.54)$ & $0.19(0.16,0.24)$ & 92 \\
\hline \multirow{2}{*}{$\mathrm{MCHxP}$} & 2018-2019 & 205 & $0.62(0.49,0.72)$ & $1.3(1.08,1.6)$ & $2.7(2.26,3.49)$ & $5.16(4.0,7.66)$ & $1.31(1.15,1.5)$ & 100 \\
\hline & 2000 & 144 & $0.93(0.63,1.41)$ & $2.63(1.96,3.16)$ & $5.11(3.97,6.27)$ & $8.1(7.17,13.97)$ & $2.01(1.66,2.43)$ & 100 \\
\hline
\end{tabular}

a LOD—limit of detection; LOD—0.013 ng/mL for all three metabolites; ${ }^{\mathrm{b}} \mathrm{NA}$ : Not calculated because detection frequency was $<60 \%$. 
The highest concentrations were for MCHxP, followed by $\mathrm{MHHpP}$ and MOHpP. However, MCHxP is not a DiHpP-specific metabolite. MCHxP can also be produced by other high-molecular-weight phthalates [23], whereas $\mathrm{MHHpP}$ and $\mathrm{MOHpP}$ are specific biomarkers for DiHpP. The GM $(95 \% \mathrm{CI})$ concentrations of $\mathrm{MOHpP}(0.19(0.16,0.24)$ vs. $0.03(0.03,0.04) \mathrm{ng} / \mathrm{mL}$ for 2000 and 2018-2019, respectively) and $\operatorname{MCHxP}(2.0(1.66,2.43)$ vs. $1.31(1.15,1.50) \mathrm{ng} / \mathrm{mL}$ for 2000 and 2018-2019, respectively) were higher in samples collected in 2000 than in 2018-2019 (Table 2). In contrast, the GM concentrations of MHHpP were higher in samples collected in 2018-2019 (0.59 (0.50, $0.70) \mathrm{ng} / \mathrm{mL})$ than in $2000(0.38(0.31,0.46) \mathrm{ng} / \mathrm{mL})$, perhaps because of differences in the formulations before and after manufacturing changes for $\mathrm{DiHpP}$ in the United States. Although, the production of $\mathrm{DiHpP}$ was discontinued in 2010 [1], the use of $\mathrm{DiHpP}$ in $\mathrm{C}_{6}-\mathrm{C}_{8}, \mathrm{C}_{7}-\mathrm{C}_{9}$, and $\mathrm{C}_{6}-\mathrm{C}_{10}$ and other commercial phthalate mixtures [1] might have contributed to exposure in later years.

As expected, the correlation analysis showed statistically significant correlations $(p<0.001)$ between the $\log _{10}$-transformed concentrations of MHHpP and MOHpP (Figure 3). The correlation was more significant in samples collected in 2000 (correlation coefficient $(\mathrm{r}),(95 \% \mathrm{CI})=0.94(0.91$, $0.95))$ than in samples collected in 2018-2019 $(r,(95 \%$ CI $)=0.59(0.49,0.67))$ (Figure 3). To explain this finding, we hypothesize that phthalate formulations used before the 2010 ending of DiHpP production in the USA may have included a larger percentage of $\mathrm{DiHpP}$ isomers with straight-chain C-backbone, where oxidation to form MOHpP can readily occur. Urinary concentrations of MHHpP also correlated with those of $\operatorname{MCHxP}(r,(95 \% \mathrm{CI})=0.87(0.83,0.91), 0.49(0.38,0.59)$ for 2000 and 2018-2019, respectively $(p<0.001)$ ) (Figure 3 ), suggesting DiHpP as the primary source for MCHxP in these samples.
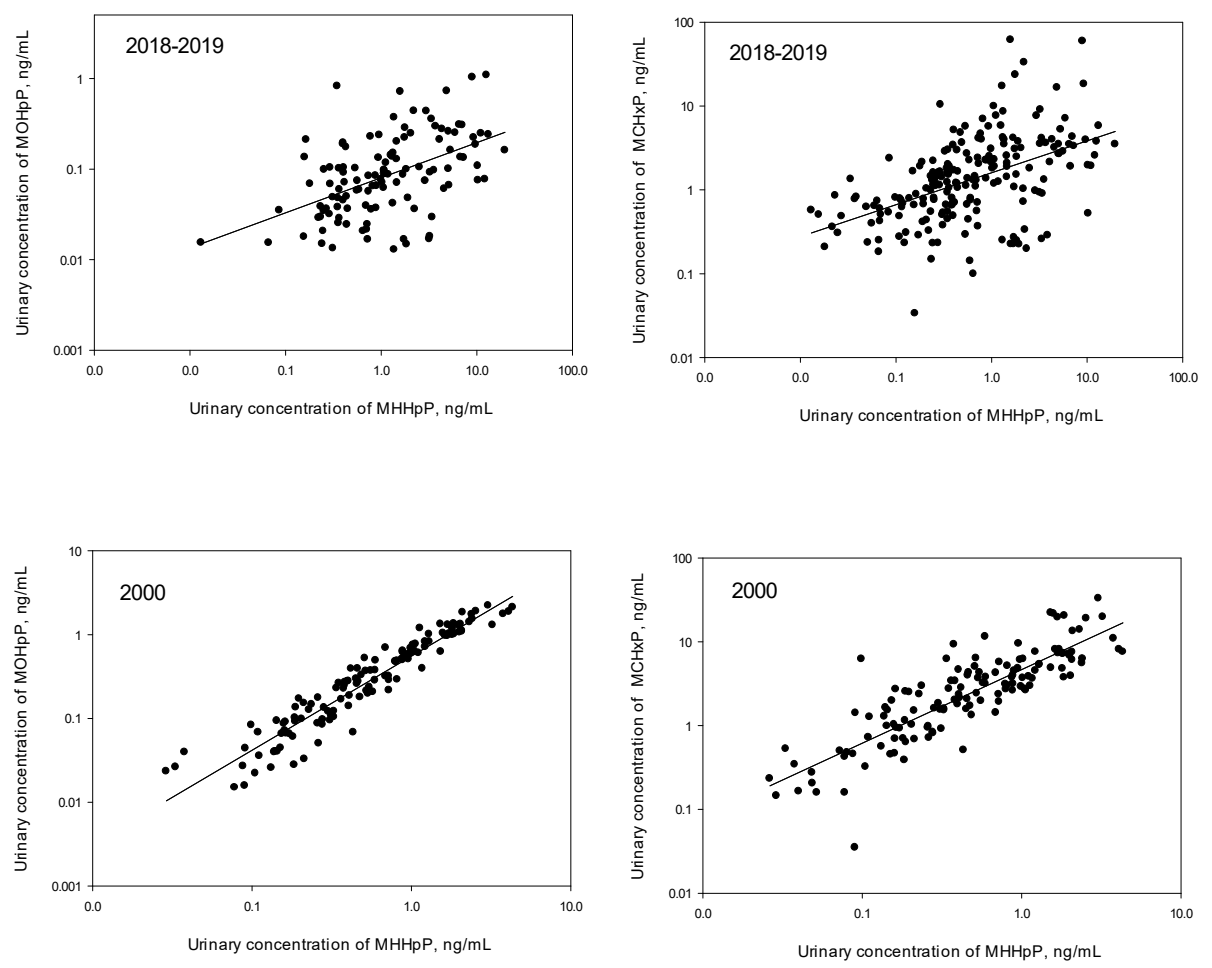

Figure 3. Correlation analyses of urinary concentrations of the metabolites of diisoheptyl phthalate (only the concentrations above the limits of detection are shown).

The detection of DiHpP metabolites among a diverse group of U.S. adults suggests exposure to DiHpP in the United States. However, the concentrations of the metabolites were lower than most other phthalate metabolites detected in human urine reported from the 2015-2016 National Health and Nutrition Examination Survey (Figure 4) [19]. These pilot data suggest that the DiHpP 
metabolites (MHHpP, $\mathrm{MOHpP}$, and $\mathrm{MCHxP}$ ) can serve as biomarkers of exposure to $\mathrm{DiHpP}$ in large-scale biomonitoring studies.

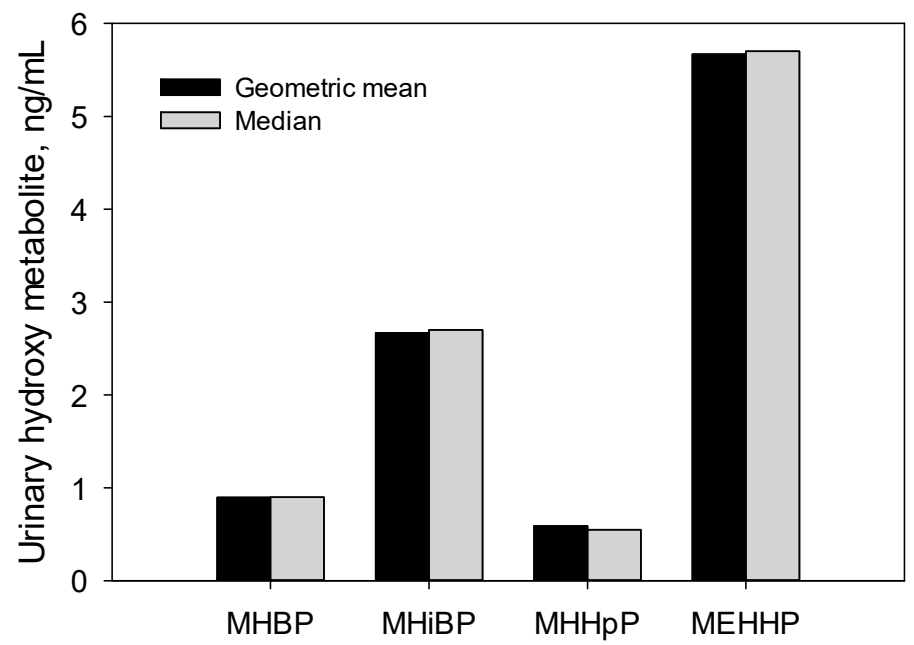

Figure 4. Geometric mean and median concentrations of the hydroxy metabolites of dibutyl phthalate (DBP), diisobutyl phthalate (DiBP), and di-2-ethylhexyl phthalate (DEHP) in urine samples from the 2015-2016 National Health and Nutrition Examination Survey (NHANES) compared with MHHpP (2018-2019 current study). Monohydroxybutyl phthalate (MHBP)-metabolite of DBP; monohydroxy-isobutyl phthalate (MHiBP)-metabolite of DiBP; mono-2-ethyl-5-hydroxyhexyl phthalate (MEHHP)-metabolite of DEHP.

\section{Disclaimer}

The use of trade names is for identification purposes only and does not constitute endorsement by the U.S. Department of Health and Human Services or the Centers for Disease Control and Prevention. The findings and conclusions in this report are those of the authors and do not necessarily represent the views of the Centers for Disease Control and Prevention.

Author Contributions: Conceptualization, methodology and validation, M.J.S.; formal data analysis, L.-Y.W., sample analysis M.J.S., J.L.P., E.O., and E.S.; investigation, writing, review and editing, M.J.S., J.C.B., and A.M.C.

Funding: This research received no external funding.

Conflicts of Interest: Authors declare no conflict of interest.

\section{References}

1. CPSC. CPSC Staff Toxicity Review of 17 Phthalates for Consideration by the Chronic Hazard Advisory Panel - 2010. Available online: https://cpsc.gov/s3fs-public/CPSCStaffToxicity17Phthalates.pdf (accessed on 10 October 2019).

2. CPSC. Toxicity Review of Diisoheptyl Phthalate (dihp). Available online: https://www.cpsc.gov/Global/ Research-and-Statistics/Technical-Reports/Chemical/Phthalates/ToxicityReviewOfDiHP.pdf (accessed on 11 September 2019).

3. Hannas, B.R.; Lambright, C.S.; Furr, J.; Howdeshell, K.L.; Wilson, V.S.; Gray, L.E. Dose-response assessment of fetal testosterone production and gene expression levels in rat testes following in utero exposure to diethylhexyl phthalate, diisobutyl phthalate, diisoheptyl phthalate, and diisononyl phthalate. Toxicol. Sci. 2011, 123, 206-216. [CrossRef] [PubMed]

4. Jin, M.L.; Dewa, Y.; Kawai, M.; Nishimura, J.; Saegusa, Y.; Kemmochi, S.; Harada, T.; Shibutani, M.; Mitsumori, K. Induction of liver preneoplastic foci in $\mathrm{f} 344$ rats subjected to 28-day oral administration of diheptyl phthalate and its in vivo genotoxic potential. Toxicology 2009, 264, 16-25. [CrossRef] [PubMed] 
5. Nakane, F.; Kunieda, M.; Shimizu, S.; Kobayashi, Y.; Akane, H.; Akie, Y.; Saito, A.; Noguchi, M.; Kadota, T.; Mitsumori, K. Twenty-six-week oral toxicity of diheptyl phthalate with special emphasis on its induction of liver proliferative lesions in male F344 rats. J. Toxicol. Sci. 2012, 37, 527-537. [CrossRef] [PubMed]

6. Lambright, C.; Howdeshell, K.; Furr, J.; Gray, L.; Wilson, V. In utero exposure to di-isoheptyl phthalate (DIHP) reduces testicular testosterone (T) production in fetal sprague dawley (SD) rats. Biol. Reprod. 2008, 547. [CrossRef]

7. McKee, R.H.; Pavkov, K.L.; Trimmer, G.W.; Keller, L.H.; Stump, D.G. An assessment of the potential developmental and reproductive toxicity of di-isoheptyl phthalate in rodents. Reprod. Toxicol. 2006, 21, 241-252. [CrossRef] [PubMed]

8. Matsushima, Y.; Onodera, H.; Mitsumori, K.; Maekawa, A.; Kurokawa, Y.; Takahashi, M. Twenty-eight day repeated dose toxicity test of dihepthyl phthalate in $\mathrm{f} 344$ rats. Eisei Shikenjo Hokoku. Bull. of Natl. Inst. Hyg. Sci. 1992, 110, 26-31.

9. Koch, H.M.; Ruther, M.; Schutze, A.; Conrad, A.; Palmke, C.; Apel, P.; Bruning, T.; Kolossa-Gehring, M. Phthalate metabolites in 24-h urine samples of the german environmental specimen bank (ESB) from 1988 to 2015 and a comparison with us nhanes data from 1999 to 2012. Int. J. Hyg. Environ. Health 2016, 220, 130-141. [CrossRef] [PubMed]

10. Koch, H.M.; Schutze, A.; Palmke, C.; Angerer, J.; Bruning, T. Metabolism of the plasticizer and phthalate substitute diisononyl-cyclohexane-1,2-dicarboxylate (DINCH (r)) in humans after single oral doses. Arch. Toxicol. 2013, 87, 799-806. [CrossRef] [PubMed]

11. Silva, M.J.; Samandar, E.; Preau, J.L.; Needham, L.L.; Calafat, A.M. Urinary oxidative metabolites of di(2-ethylhexyl) phthalate in humans. Toxicology 2006, 219, 22-32. [CrossRef] [PubMed]

12. Calafat, A.M.; Wong, L.Y.; Silva, M.J.; Samandar, E.; Preau, J.L.; Jia, L.T.; Needham, L.L. Selecting adequate exposure biomarkers of diisononyl and diisodecyl phthalates: Data from the 2005-2006 national health and nutrition examination survey. Environ. Health Perspect. 2011, 119, 50-55. [CrossRef] [PubMed]

13. Schutze, A.; Gries, W.; Kolossa-Gehring, M.; Apel, P.; Schroter-Kermani, C.; Fiddicke, U.; Leng, G.; Bruning, T.; Koch, H.M. Bis-(2-propylheptyl)phthalate (DPHP) metabolites emerging in $24 \mathrm{~h}$ urine samples from the german environmental specimen bank (1999-2012). Int. J. Hyg. Environ. Health 2015, 218, 559-563. [CrossRef] [PubMed]

14. Park, C.; Hwang, M.; Baek, Y.; Jung, S.; Lee, Y.; Paek, D.; Choi, K. Urinary phthalate metabolite and bisphenol a levels in the korean adult population in association with sociodemographic and behavioral characteristics: Korean national environmental health survey (KoNEHS) 2012-2014. Int. J. Hyg. Environ. Health 2019, 222, 903-910. [CrossRef] [PubMed]

15. Saravanabhavan, G.; Guay, M.; Langlois, É.; Giroux, S.; Murray, J.; Haines, D. Biomonitoring of phthalate metabolites in the canadian population through the canadian health measures survey (2007-2009). Int. J. Hyg. Environ. Health 2013, 216, 652-661. [CrossRef] [PubMed]

16. Sato, M.; Adachi, T.; Tanaka, A.; Yamaha, T. Biochemical-studies on phthalic esters. 4. Metabolism of diheptyl phthalate in rats. Drug Metab. Dispos. 1984, 12, 517-522. [PubMed]

17. Silva, M.J.; Samandar, E.; Preau, J.L.; Reidy, J.A.; Needham, L.L.; Calafat, A.M. Quantification of 22 phthalate metabolites in human urine. J. Chromatogr. B 2007, 860, 106-112. [CrossRef] [PubMed]

18. Silva, M.J.; Jia, T.; Samandar, E.; Preau, J.L.; Calafat, A.M. Environmental exposure to the plasticizer 1,2-cyclohexane dicarboxylic acid, diisononyl ester (DINCH) in us adults (2000-2012). Environ. Res. 2013, 126, 159-163. [CrossRef] [PubMed]

19. National Center for Environmental Health; Division of Laboratory Sciences. National Report on Human Exposure to Environmental Chemicals; Department of Health and Human Services Centers for Disease Control and Prevention: Atlanta, GA, USA, 2019.

20. Hornung, R.W.; Reed, L.D. Estimation of average concentration in the presence of nondetectable values. Appl. Occup. Environ. Hyg. 1990, 5, 46-51. [CrossRef]

21. Koch, H.M.; Muller, J.; Angerer, J. Determination of secondary, oxidised di-iso-nonylphthalate (DINP) metabolites in human urine representative for the exposure to commercial dinp plasticizers. J. Chromatogr. B 2007, 847, 114-125. [CrossRef] [PubMed]

22. Silva, M.J.; Bontke, T.W.; Calafat, A.M.; Ye, X. Identification of potential biomarkers of exposure to diundecyl phthalate. Environ. Res. 2016, 148, 137-143. [CrossRef] [PubMed] 
23. Kato, K.; Silva, M.J.; Wolf, C.; Gray, L.E.; Needham, L.L.; Calafat, A.M. Urinary metabolites of diisodecyl phthalate in rats. Toxicology 2007, 236, 114-122. [CrossRef] [PubMed]

(C) 2019 by the authors. Licensee MDPI, Basel, Switzerland. This article is an open access article distributed under the terms and conditions of the Creative Commons Attribution (CC BY) license (http://creativecommons.org/licenses/by/4.0/). 